\title{
On Maximum Field Components in the Focal Point of a Lens.
}

\author{
H.P. Urbach*a, S.F. Pereira ${ }^{\mathrm{a},}$, D.J. Broer ${ }^{\mathrm{b}}$ \\ ${ }^{a}$ Optics Research Group, Dept. of Imaging Science, Delft University of Technology, Delft, P.O. Box \\ 5046, 2600GA Delft, The Netherlands; \\ ${ }^{\mathrm{b}}$ Polymers in Advanced Systems Group, Faculty of Chemical Engineering and Chemistry, \\ Eindhoven University of Technology, P.O. Box 513, 5600MB Eindhoven, The Netherlands.
}

\begin{abstract}
We determine field distributions in the pupil of a high NA lens, that give, for a given power incident on the lens, the maximum electric field amplitude in focus in a specific direction. We consider in particular the cases of maximum longitudinal and maximum transverse components. The distribution of the maximum longitudinal component in the focal plane is narrower than that of the focused Airy spot and hence can give higher resolution in imaging.
\end{abstract}

Keywords: High NA, beam shaping, optimization, longitudinal polarization

\section{INTRODUCTION}

A linear polarized plane wave that is focused by a diffraction limited lens, gives an intensity distribution in the focal plane which in the paraxial optics is called the Airy pattern. When the numerical aperture exceeds 0.6, rotation of polarization can not be neglected and vector diffraction theory must be applied. The accepted model to compute the field distribution near focus has been derived by Ignatowsky ${ }^{1}$ and Richards and Wolf ${ }^{2}$. The intensity distribution obtained in the focal plane with this theory when a linear polarized plane wave is focused, is called the vector Airy pattern.

Today almost any pupil distribution in the visible can be realized using spatial light modulators. Recently, spots with large longitudinal component have raised a lot of interest. These fields can be realized by focusing a radial polarized pupil field Dorn ${ }^{3}$, Quabis ${ }^{4}$, Sheppard ${ }^{5}$. It turns out that the distribution of the longitudinal component in the focal plane is narrower than the vector Airy spot. When a recording medium would be used that is sensitive to only the longitudinal electric field component, images with higher resolution would be obtained. Photo-resists that have this anisotropic response can be realized by embedding a photo-initiator in a self-organizing liquid crystal monomer host.

The radial polarized pupil fields that were used in previous research, yield a relatively high longitudinal component in focus, but not the largest possible. It is thus of interest to determine the amplitude distribution in the pupil for which, for given power incident on the lens pupil, the longitudinal component is maximum. More generally, we consider the problem of determining the pupil field (i.e. amplitude, phase and polarization as function of the pupil coordinates), for which for given total incident power, some component of the electric field at focus has maximum amplitude. It turns out that the optimum field distributions can be determined analytically. Apart from potential applications in biosensors, photolithography, optical disk mastering etc., these results are also of interest from the point of view of theoretical optics.

*h.p.urbach@tudelft.nl phone $\quad+31152789406 ; \quad$ fax $\quad+31152788105 ; \quad$ www.optica.tn.tudelft.nl 


\section{OPTIMIZATION OF THE FIELDS IN THE PUPIL}

\subsection{The pupil field and the plane wave expansion in image space.}

The plane wave expansion in image space is

$$
\vec{E}(\vec{r})=\iint \vec{A}\left(k_{x}, k_{y}\right) \exp (i \vec{k} \vec{r}) d k_{x} d k_{y}
$$

where the integration is restricted to the disc $k_{x}{ }^{2}+k_{y}{ }^{2}<k N A$. By using spherical coordinates in $k$-space:

$$
\begin{aligned}
& \widehat{k}=\sin \alpha \cos \beta \cdot \widehat{x}+\sin \alpha \sin \beta \cdot \widehat{y}+\cos \alpha \cdot \widehat{z} \\
& \hat{\alpha}=\cos \alpha \cos \beta \cdot \widehat{x}+\cos \alpha \sin \beta \cdot \hat{y}-\sin \alpha \cdot \widehat{z} \\
& \widehat{\beta}=-\sin \beta \cdot \widehat{x}+\cos \beta \cdot \widehat{y}
\end{aligned}
$$

with $\alpha$ and $\beta$ the polar and azimuth angles of the wave vector, respectively, the plane wave expansion can be rewritten as integral over $\alpha$ and $\beta$ :

$$
\vec{E}(\vec{r})=(k / 2 \pi)^{2} \int_{0}^{\alpha_{\max }} \int_{0}^{2 \pi}\left[A_{\alpha}(\alpha, \beta) \hat{\alpha}+A_{\beta}(\alpha, \beta) \hat{\beta}\right] \cos \alpha \sin \alpha \exp (i \vec{k} . \vec{r}) d \alpha d \beta
$$

where $\alpha_{\max }=\arcsin (N A)$. According to the theory of Ignatowsky ${ }^{1}$ and Richards and Wolf $^{2}$, the polar and azimuthal components of the plane wave amplitudes are related to the components of the field in the pupil on the basis of polar pupil coordinates: $\widehat{\rho}, \widehat{\varphi}$ :

$$
\vec{E}^{p}(\rho, \varphi)=E_{\rho}^{p}(\rho, \varphi) \hat{\rho}+E_{\varphi}^{p}(\rho, \varphi) \hat{\varphi}
$$

by the following equations:

$$
\begin{aligned}
& A_{\alpha}(\alpha, \beta)=\left(\lambda \rho_{\max } / N A \sqrt{\cos \alpha}\right) E_{\rho}^{p}(\rho, \varphi) \\
& A_{\beta}(\alpha, \beta)=\left(\lambda \rho_{\max } / N A \sqrt{\cos \alpha}\right) E_{\varphi}^{p}(\rho, \varphi) \\
& \rho=\rho_{\max } \sin \alpha / N A, \\
& \varphi=\beta+\pi .
\end{aligned}
$$

where $\rho_{\max }$ is the radius of the pupil. Let $\widehat{v}$ be a unit vector and let the coordinate system be chosen such that the rigin coincides with the centre of the Gaussian reference sphere, i.e. with the focal point. Then the projection along $\widehat{v}$ of the electric field vector at the focal point is:

$$
\vec{E}(\overrightarrow{0}) \cdot \hat{v}=(k / 2 \pi)^{2} \int_{0}^{\alpha_{\max }} \int_{0}^{2 \pi}\left[A_{\alpha}(\alpha, \beta) v_{\alpha}+A_{\beta}(\alpha, \beta) v_{\beta}\right] \cos \alpha \sin \alpha d \alpha d \beta
$$


By substitution of the first two equations of (3) for $A_{\alpha}$ and $A_{\beta}, \vec{E}(\overrightarrow{0}) \cdot \widehat{v}$ becomes a functional of the pupil field components $E_{\rho}^{p}$ and $E_{\varphi}^{p}$. The total power incident on the pupil is, apart from a constant factor:

$$
P\left(E_{\rho}^{p}, E_{\varphi}^{p}\right)=\int_{0}^{2 \pi} \int_{0}^{\rho_{\max }}\left[\left.E_{\rho}^{p}(\rho, \varphi)\right|^{2}+\left|E_{\varphi}^{p}(\rho, \varphi)\right|^{2}\right] \rho d \rho d \varphi
$$

\subsection{The optimization problem}

Formula (4) gives the electric field amplitude along the $\widehat{v}$-direction at time $t=0$. We may assume that this amplitude is real. The optimization problem is then to determine the pupil field components $E_{\rho}^{p}$ and $E_{\varphi}^{p}$ such that $\vec{E}(\overrightarrow{0}) \cdot \hat{v}$ is maximum under the condition that the mean power incident on the pupil is given, i.e.

Maximize $\vec{E}(\overrightarrow{0}) \cdot \hat{v} \quad$ under the constraint: $P\left(E_{\rho}^{p}, E_{\varphi}^{p}\right)=P_{0}$,

for some given power $P_{0}$. It turns out that the optimum pupil field corresponding to every choice of $\widehat{v}$ can be computed analytically, i.e. we can obtain closed formulae for the optimum pupil field. After a long but elementary computation, one finds

$$
\begin{aligned}
& E_{\rho}^{p}(\rho, \varphi)=\sqrt{1-\left(N A \rho / \rho_{\max }\right)^{2}} \cdot v_{\rho}+\frac{N A \rho / \rho_{\max }}{\left[1-\left(N A \rho / \rho_{\max }\right)^{2}\right]^{1 / 4}} v_{z} \\
& E_{\varphi}^{p}(\rho, \varphi)=\frac{1}{\left[1-\left(N A \rho / \rho_{\max }{ }^{2}\right]^{1 / 4}\right.} v_{\varphi}
\end{aligned}
$$

In these formulae, $v_{\rho}$ and $v_{z}$ are the coordinates of $\widehat{v}$ on the basis of cylindrical coordinates. By using Eqns. (3), the plane wave amplitudes of the optimum field can be determined. Substitution of these into (1) then gives the electric field distribution near the focal plane of the field for which the $\vec{E}(\overrightarrow{0}) \cdot \hat{v}$ component is maximum.

\subsection{Results and discussion}

In all examples, the numerical aperture of the lens is 0.9 . When $\widehat{v}=\widehat{z}$, the component in focus that is maximized is the longitudinal component. It follows from (7) and (8) that the optimum electric field in the pupil then is indeed radial polarized with amplitude that increases with radius towards the rim of the aperture. The electric field in the pupil is shown at the upper left of Fig. 1 in Fig. 2, the distribution of the squared amplitude of the optimum longitudinal component in the focal plane is compared to the intensity distribution of the Airy spot. The width of the longitudinal spot is found to be $20 \%$ less than that of the Airy spot for numerical aperture of 0.9 Note that the Airy spot is elliptically shaped. 
If we choose the direction of $\hat{v}$ transverse to the optical axis, e.g. parallel to $\hat{x}$, the transverse electric field is maximized. The corresponding optimum pupil field is shown at the lower right in Fig. 1. It is seen that the field in the pupil is very similar to that of an $x$-polarized plane wave, but that it is not identical to it. Hence the Airy spot does not give the maximum transverse electric field amplitude in focus, although it almost does. The other pupil fields shown in Fig. 1 are the solutions for which the electric field component in focus which makes an angle of $30^{\circ}$, (upper right) and $60^{\circ}$ (lower left) with the optical axis is maximum.
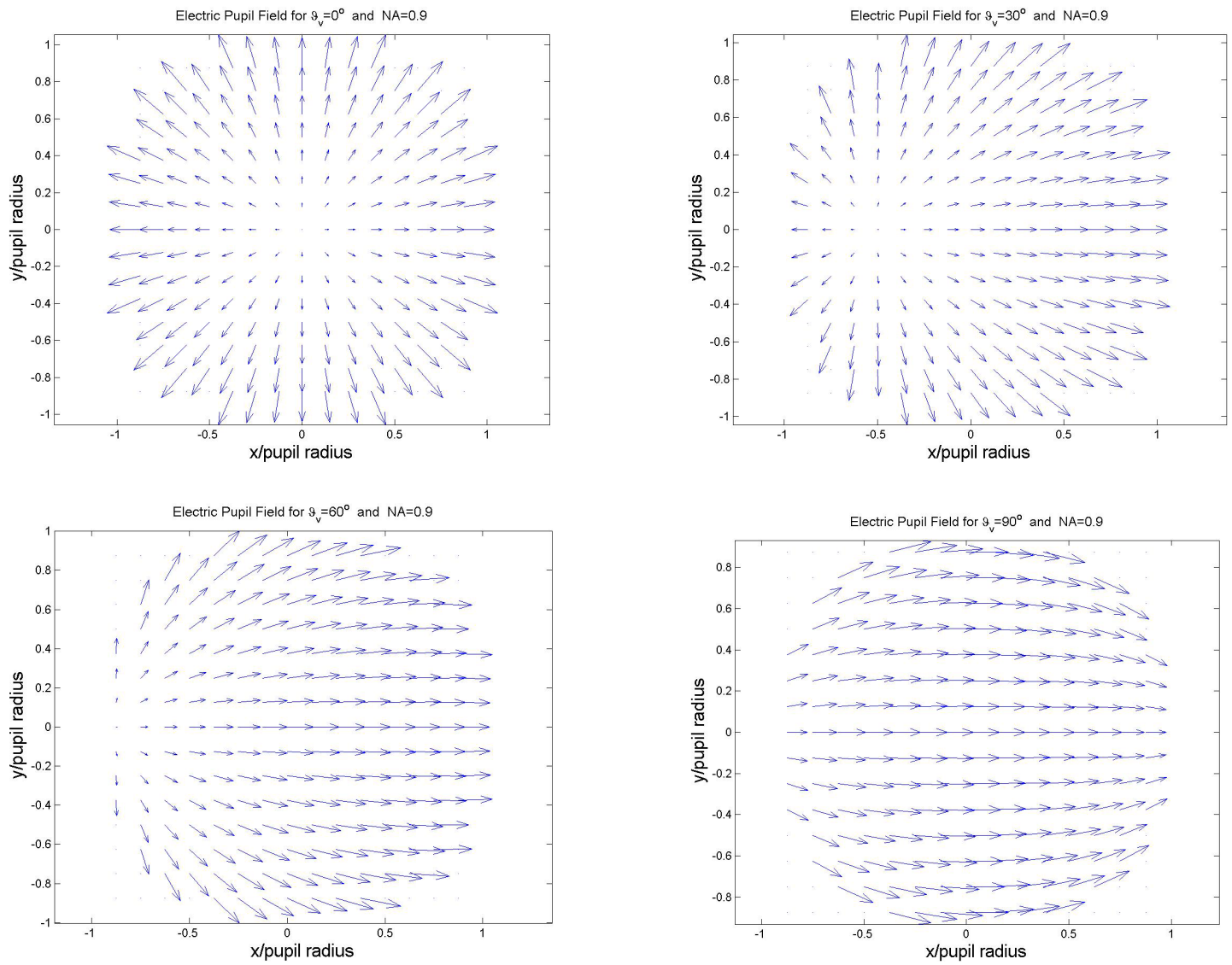

Figure 1. Optimum electric field in the pupil of the lens for the case that $\widehat{v}=\widehat{z}$ (longitudinal component, upper left), $\widehat{v}$ makes an angle of $30^{\circ}$ with the $z$-axis (upper right), $\quad \hat{v}$ makes an angle of $60^{\circ}$ with the $z$-axis (lower left) and $\widehat{v}=\hat{x}$ (transverse component, lower right).

\section{POLARIZATION SENSITIVE PHOTORESIST}

To use the smaller spot width of the longitudinal component of the electric field to gain a better resolution, it is essential that the recording medium is sensitive to only the longitudinal component. Although exactly in the focal point the longitudinal component is the only nonzero component, the transverse components are nonzero in a neighborhood of the focal point. In fact, the transverse field is rather broad and its maximum is also considerable so that the total intensity distribution in the focal plane is even wider than that of the Airy spot. Hence, an anisotropic recording medium is essential. 
The recording medium that we propose is based on a self-organizing monomer system that can be converted from the soluble low molar mass resin into an insoluble cross linked polymer by light-induced reactions using a dichroic photoinitiator. This principle is schematically shown in Fig. 3. The negative resist will have a preferential monomer orientation perpendicular to the substrate. Essential in this principle is a new photo-sensitive material that has a preferred rod-like conformation and a discrete transition moment along the long axis of the molecular. A recently developed material is shown in Fig. 4. The same figure shows that this photo-initiator has a dichroic ratio, being the ratio of absorption for light with their electrical field vectors parallel and perpendicular respectively, of around 6.
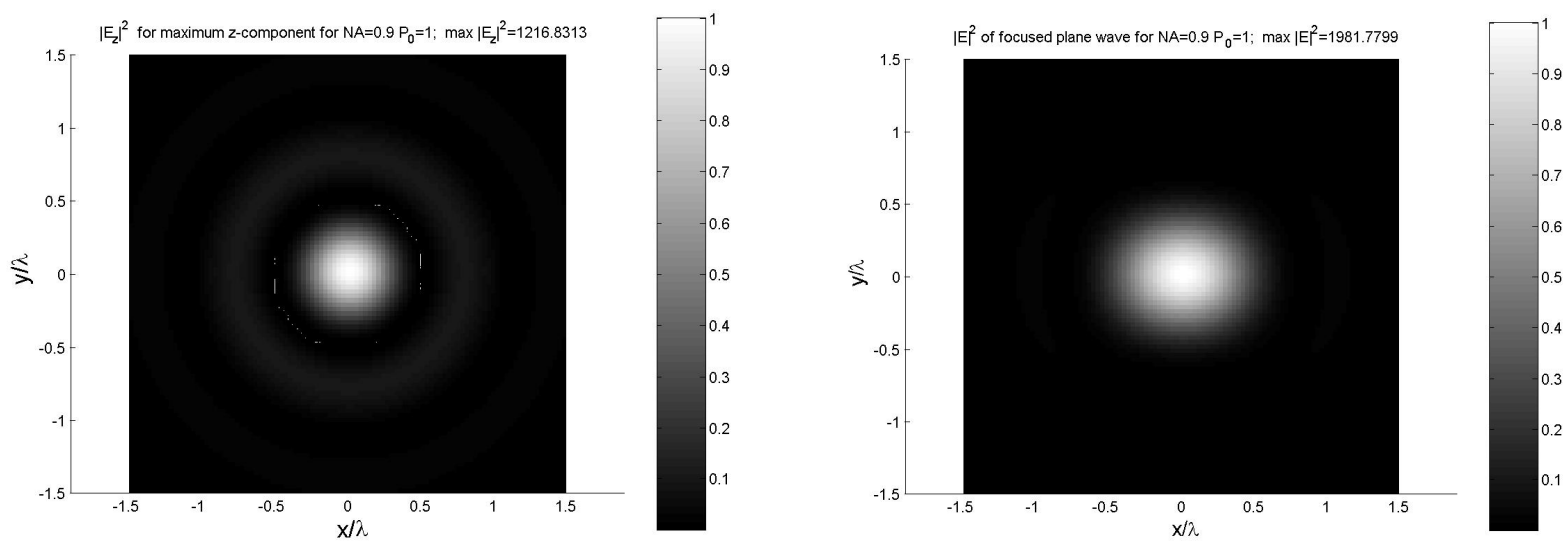

Figure 2. Distribution of the squared amplitude of the optimized longitudinal electric field component in the focal plane (left) and the intensity distribution of the Airy spot in the focal plane (right). In both cases the numerical aperture is 0.9 .
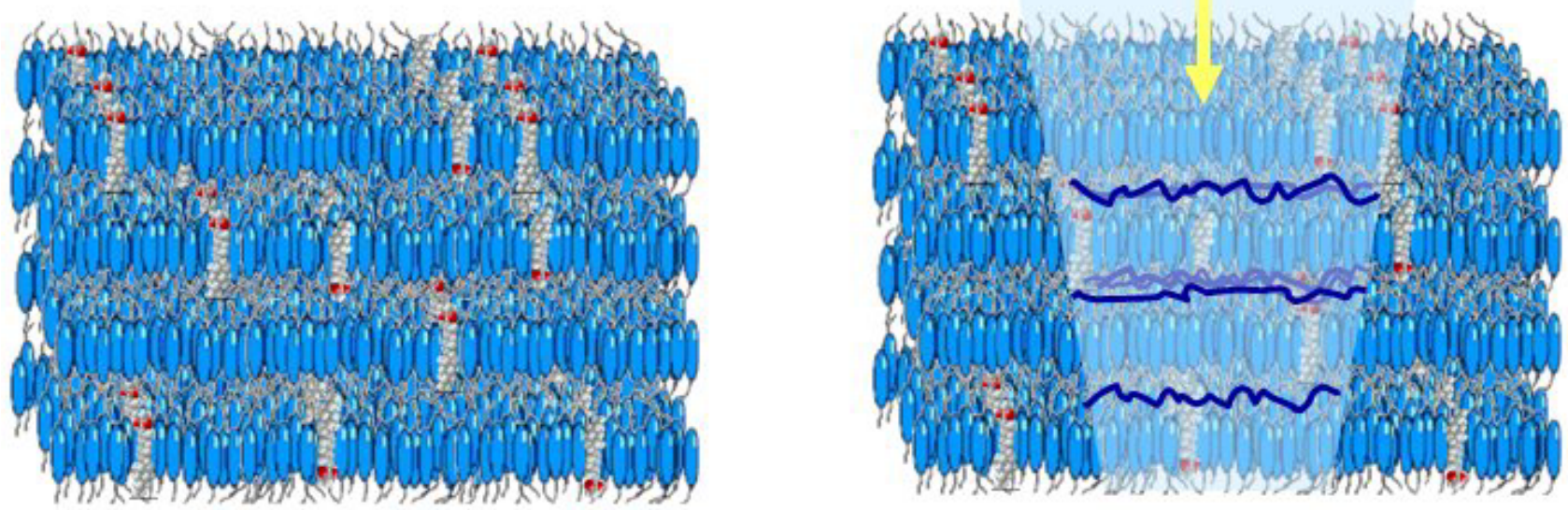

Figure 3. Principle of the polarization-sensitive resist. A rod-like shaped photosensitive initiator is aligned parallel to the propagation direction of the incoming light by dissolving it in a liquid crystal monomer.

The ratio of absorption of 6 , corresponds to an order parameter of the initiator of 0.6 , similar to that of the nematic host. The dichroic photo-initiator adapts the orientation of its self-organizing monomer host such that its transition dipole 
moment will also be perpendicular to the substrate, i.e. parallel to longitudinal direction. As a result it will have low absorption for the transverse electrical field components and a high absorption for longitudinal electrical component of traversing light. Self-organizing monomers known to align themselves with a high degree of monolithic order preferably have plurality of mesophases, with a nematic phase at elevated temperatures and a smectic-B phase at moderate temperatures ${ }^{6,7,9}$. Polymerization will take place in the smectic-B phase. Rather than the nematic phase (Fig.. 4) the smectic-B phase is chosen, as it combines a high order parameter, and thereby a high lithographic contrast, with probably sufficient mobility for polymerization and cross-linking to occur. The dichroic ratio is then expected to be even more than 50. This has been demonstrated ${ }^{6,7,9}$ for dye systems embedded in smectic-B hosts as shown in Fig. 5 . Preferably, the molecular order as imposed by the smectic-B phase is locked in a glassy state at room temperature to facilitate processing and manipulation of the layers.
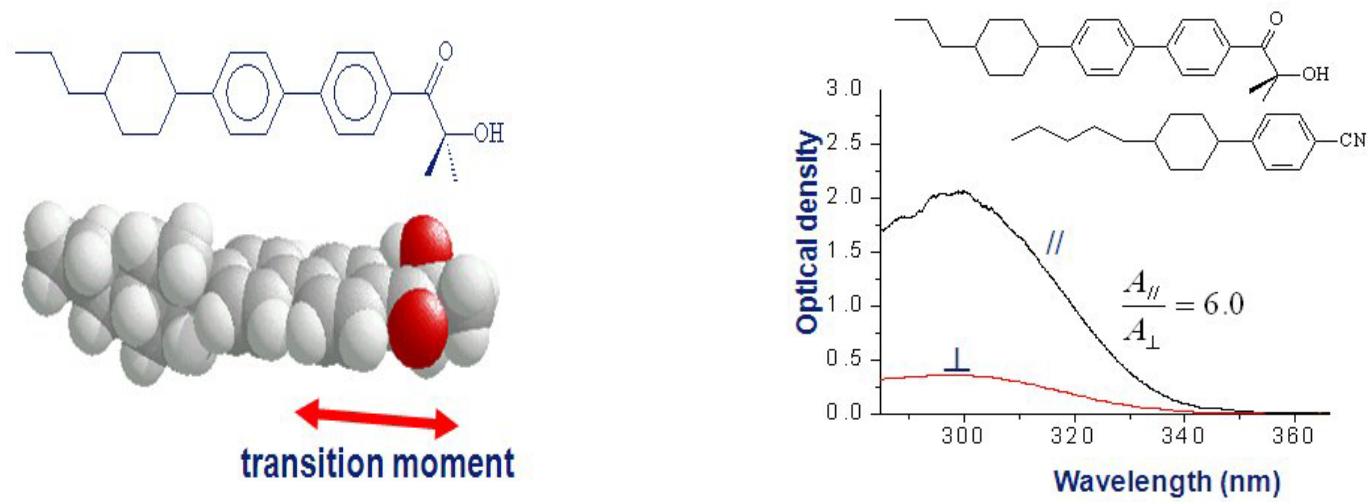

Figure 4. Left: example of a dichroic photosensitive initiator. The photosensitive component has its transition moment parallel to the longitudinal axis. Right: absorbance of the dichroic photo-initiator, dissolved in a nematic host, with its axis parallel or perpendicular to the polarization of light.
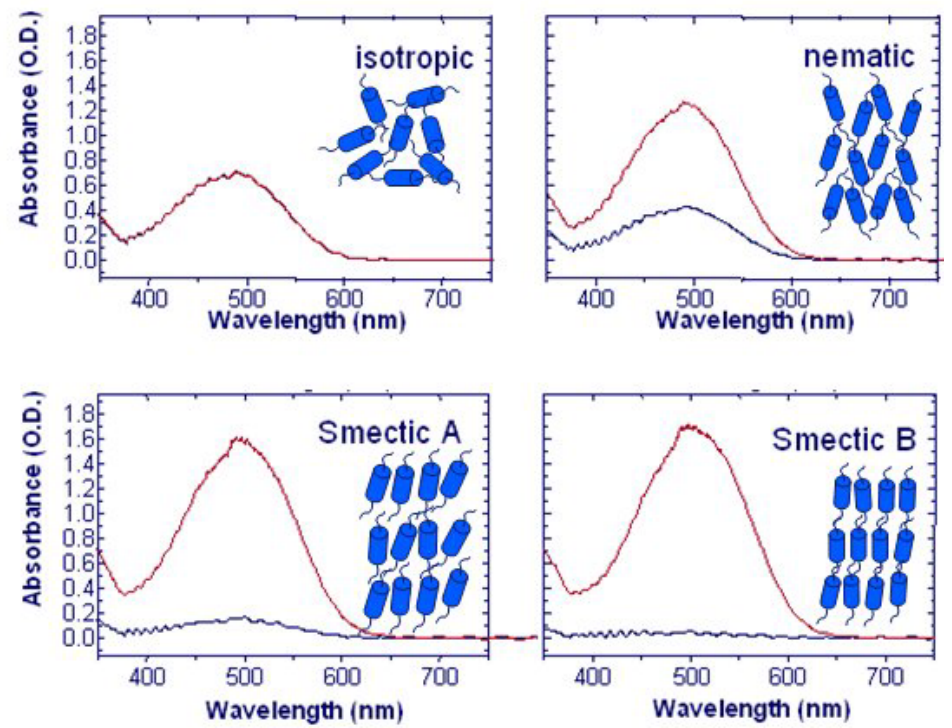

Figure 5. Enhancement of the dichroic ratio of a chromophore molecule, exemplary for the initiator system, in liquid crystal hosts with different degree of order, ranging from isotropic to nematic, to smectic A and smectic B. 


\section{REFERENCES}

1. V. S. Ignatowsky, Trans. Opt. Inst. Petrograd, Vol. I, paper IV and V (1919).

2. B. Richards and E. Wolf, "Electromagnetic diffraction in optical systems II. Structure of the image field in an aplanatic system", Proc. R. Soc. London Ser. A 253, 358-379 (1959)..

3. R. Dorn, S. Quabis and G. Leuchs, "Sharper focus for a radially polarized beam", Phys. ev.Lett., 91, Issue 23, 233901 (2003).

4. S. Quabis, R. Dorn, M. Eberler, O. Gl"ockl and G. Leuchs, "Focusing light to a tighter spot", Optics Communications, 179, 1-7, .(2000).

5. C.J.R. Sheppard and P. Torok, "Electromagnetic field in the focal region of an electric dipole wave", Optik, 104, No. 4, 175-177, (1997).

6. E. Peeters, J. Lub, J.A.M. Steenbakkers J.A.M, D.J. Broer, "High-Contrast Thin-Film Polarizers by photocrosslinking of Smectic Guest-Host Systems", Adv. Mat. 18, 2412-2417 (2006).

7. B.M.I. van der Zande, J. Steenbakkers, J. Lub, C.M. Leewis and D.J. Broer, "Mass transport phenomena during lithographic polymerization of nematic monomers monitored with intrferometry", J. Appl. Phys.,97, No 12, (2005).

8. C. Sanchez, B.J. de Gans, D.A. Kozodaev, A. Alekseev, M.J. Escuti, C. van Heesch, D.L. Beke, U.S. Schubert, C.W.M. Bastiaansen, D.J. Broer, "Polymerization-induced Diffusion as a Tool to Generate Periodic Relief Structures: a Combinatorial Study", Proc. SPIE, 6136, 61360H/1-61360H/12, (2006).

9. S.R. Kennedy, J.C. Sit, D.J. Broer, M.J. Brett, "Optical Activity of Chiral Thin Film and Liquid Crystal Hybrids", Liq. Cryst., 28, No. 12, 1799-1803, (2003). 